\title{
The evolution and expression of panarthropod frizzled genes
}

\section{OPEN ACCESS}

Edited by:

Angelika Stollewerk,

Queen Mary University of London, UK

Reviewed by:

Lucas Leclere,

University Pierre et Marie Curie,

France

Ildiko M. L. Somorjai,

University of St. Andrews, UK

*Correspondence:

Alistair P. McGregor,

Department of Biological and Medical

Sciences, Oxford Brookes University,

Gipsy Lane, Oxford OX3 OBP, UK

amcgregor@brookes.ac.uk;

Maarten Hilbrant,

Institute for Developmental Biology,

University of Cologne, Zülpicher

Straße 47b, 50674 Cologne, Germany

m.hilbrant@uni-koeln.de

${ }^{\dagger}$ Present Address:

Maarten Hilbrant,

Institute for Developmental Biology,

University of Cologne, Cologne,

Germany

${ }^{\ddagger}$ These authors have contributed equally to this work

Specialty section:

This article was submitted to

Evolutionary Developmental Biology,

a section of the journal

Frontiers in Ecology and Evolution

Received: 12 June 2015 Accepted: 03 August 2015 Published: 21 August 2015

Citation: Janssen $R$, Schönauer A, Weber $M$, Turetzek N, Hogvall M, Goss GE, Patel

NH, McGregor AP and Hilbrant M

(2015) The evolution and expression of panarthropod frizzled genes.

Front. Ecol. Evol. 3:96.

doi: 10.3389/fevo.2015.00096

\section{Ralf Janssen ${ }^{1 \neq}$, Anna Schönauer ${ }^{2 \ddagger}$, Melanie Weber ${ }^{2,3}$, Natascha Turetzek ${ }^{4}$, Mattias Hogvall ${ }^{1}$, Georgina E. Goss ${ }^{2}$, Neel H. Patel ${ }^{2}$, Alistair P. McGregor ${ }^{2 *}$ and Maarten Hilbrant ${ }^{2 * t}$}

\begin{abstract}
${ }^{1}$ Department of Earth Sciences, Palaeobiology, Uppsala University, Uppsala, Sweden, ${ }^{2}$ Department of Biological and Medical Sciences, Oxford Brookes University, Oxford, UK, ${ }^{3}$ Department of Neurobiology, University of Vienna, Vienna, Austria, ${ }^{4}$ Department of Developmental Biology, GZMB, Johann-Friedrich-Blumenbach-Institute for Zoology and Anthropology, Georg-August-University Göttingen, Göttingen, Germany
\end{abstract}

Wnt signaling regulates many important processes during metazoan development. It has been shown that Wnt ligands represent an ancient and diverse family of proteins that likely function in complex signaling landscapes to induce target cells via receptors including those of the Frizzled (Fz) family. The four subfamilies of $F z$ receptors also evolved early in metazoan evolution. To date, Fz receptors have been characterized mainly in mammals, the nematode Caenorhabditis elegans and insects such as Drosophila melanogaster. To compare these findings with other metazoans, we explored the repertoire of $f z$ genes in three panarthropod species: Parasteatoda tepidariorum, Glomeris marginata, and Euperipatoides kanangrensis, representing the Chelicerata, Myriapoda, and Onychophora, respectively. We found that these three diverse panarthropods each have four $f z$ genes, with representatives of all four metazoan fz subfamilies found in Glomeris and Euperipatoides, while Parasteatoda does not have a $f z 3$ gene, but has two $f z 4$ paralogs. Furthermore, we characterize the expression patterns of all the $f z$ genes among these animals. Our results exemplify the evolutionary diversity of Fz receptors and reveals conserved and divergent aspects of their protein sequences and expression patterns among panarthropods, thus providing new insights into the evolution of Wnt signaling more generally.

Keywords: Wnt signaling, Frizzled receptors, development, evolution, gene expression

\section{Introduction}

In metazoans most key developmental processes from cell division to differentiation and death are regulated by Wnt signaling (Logan and Nusse, 2004). Wnt ligands bind to cell surface receptors including those encoded by frizzled $(f z)$ genes (Bhanot et al., 1996; Dann et al., 2001). Fz proteins are seven-pass transmembrane domain receptors with an $\mathrm{N}$-terminal cysteine rich domain (CRD), which regulates the specificity of interactions with Wnt ligands, and typically a C-terminal KTxxxW motif that is required for downstream interactions with the intracellular protein Disheveled (Umbhauer et al., 2000; Wong et al., 2003; Huang and Klein, 2004; Tauriello et al., 2012). As well as their roles in the canonical Wnt pathway, Fz proteins are also involved in the planar cell polarity pathway, so called non-canonical Wnt pathways (including the Wnt/calcium pathway), and can be bound by other (i.e., non-Wnt) ligands (Veeman et al., 2003; Huang and Klein, 2004; van Amerongen and Nusse, 2009). 
It has been shown that two $f z$ genes found in sponges likely gave rize to four $f z$ genes in the common ancestor of bilaterians: FZD I, II, III, and IV (Schenkelaars et al., 2015). Lineage specific expansions followed, for example in mammals $($ FZD I = Fz1/2/3/6/7; FZD II = Fz5/8; FZD III = Fz9/10; FZD $\mathrm{IV}=\mathrm{Fz} 4)$. Moreover, metazoans had an ancestral repertoire of 13 subfamilies of Wnt ligands, although there have been lineage specific duplications, for example in vertebrates, and the loss of particular ligands as seen in nematodes and insects (Prud'homme et al., 2002; van Amerongen and Nusse, 2009; Cho et al., 2010; Janssen et al., 2010; Schenkelaars et al., 2015). This considerable array of possible combinations of ligands and receptors likely underlies the diversity of roles played by Wnt signaling during metazoan development. Furthermore, it is thought that for many developmental processes landscapes of combinations of Wnt ligands and receptors are employed to achieve a robust outcome (van Amerongen and Nusse, 2009).

Fully understanding the evolution and function of Wnt signaling in metazoans requires characterizing the expression of key components such as the receptors and ligands in a wide diversity of lineages. In the arthropods and related phyla, the expression of Wnt ligand genes has been described in a range of species (Cho et al., 2010; Janssen et al., 2010; Murat et al., 2010; Hogvall et al., 2014; Janssen and Posnien, 2014), however the expression of the $f z$ genes during embryogenesis has only been fully characterized in the insects Drosophila melanogaster and Tribolium castaneum (Bhanot et al., 1996; Kennerdell and Carthew, 1998; Beermann et al., 2011). Such studies suggest that the four ancient $f z$ families were found in the common ancestor of arthropods but that there have also been lineage specific losses, like the loss of $f z 3$ in Tribolium (Beermann et al., 2011). In addition, analysis of $f z$ expression and function in these insects clearly shows that many aspects of development employ multiple receptors, as well as more than one ligand (Bolognesi et al., 2008; Janssen et al., 2010; Beermann et al., 2011).

To provide a more comprehensive description of $f z$ expression and evolution among arthropods, here we report our analysis of $f z$ genes and their expression in representatives of the Myriapoda and Chelicerata, the pill millipede, Glomeris marginata (Dohle, 1964), and the common house spider, Parasteatoda tepidariorum (Hilbrant et al., 2012), respectively. Moreover, to provide a broader perspective to $f z$ expression in the panarthropods (Onychophora + Arthropoda, Nielsen, 1995; and see Edgecombe and Legg, 2014 for a recent discussion), we also characterized the repertoire and function of these genes in the onychophoran Euperipatoides kanangrensis (Eriksson and Tait, 2012; Janssen and Budd, 2013).

Our study confirms that the common ancestor of the panarthropods contained copies of the four ancestral $\mathrm{Fz}$ subfamilies, but that some subfamilies have also been subject to loss or duplication in chelicerates. Analysis of the expression patterns of the four subfamilies of Fz genes during the embryonic development of Euperipatoides, Glomeris, and Parasteatoda reveals both similarities and differences between these lineages. Our characterization of $\mathrm{Fz}$ gene expression patterns with respect to the known patterns of Wnt ligands in these animals also helps to better understand the Wnt landscapes that regulate their development.

\section{Methods}

\section{Sequences and Phylogenetic Analysis}

$\mathrm{Fz}$ sequences were recovered from published resources: Euperipatoides kanangrensis (Janssen and Budd, 2013); Glomeris marginata (Janssen and Posnien, 2014); Parasteatoda tepidariorum (Posnien et al., 2014); Mesobuthus martensii (Cao et al., 2013); Ixodes scapularis (Lawson et al., 2009): Tribolium castaneum (Richards et al., 2008); Zootermopsis nevadensis (Terrapon et al., 2014); Strigamia maritima (Chipman et al., 2014). Pholcus phalangioides sequences were recovered from a de novo assembled transcriptome, generated from a combination of different embryonic stages and three larval tissues including legs, the prosoma, and the opisthosoma. Total RNA for larval tissue was extracted using the ZR Tissue \& Insect RNA MicroPrep ${ }^{\text {TM }}$ Kit (Zymo Research) and total RNA extraction of embryonic samples was performed using Trizol. The library for Illumina sequencing was generated using the standard protocol of the TruSeq RNA Sample Preparation Kit (v2, Illumina). All samples were pooled and sequenced on one lane of an Illumina Hiseq2000 sequencer, which resulted in $472,770,758$ paired-end reads of $100 \mathrm{bp}$. The reads were quality trimmed and filtered as previously described (Posnien et al., 2014). All high quality reads were assembled into 444,103 transcripts using the de novo transcriptome assembler Trinity (version r2013_11_10, Grabherr et al., 2011). The GC content of this preliminary de novo assembly is $37.27 \%$. The N50 is 1517 bp with a median transcript length of $284 \mathrm{bp}$ and an average transcript length of $670 \mathrm{bp}$.

To infer phylogenetic relationships, predicted $\mathrm{Fz}$ amino acid sequences were first aligned using MUSCLE (Edgar, 2004; Sievers et al., 2011) after which poorly aligned sites were masked using Gblocks v. 0.91b (Castresana, 2000) with relaxed selection criteria, both as implemented in Seaview v. 4.5.3 (Gouy et al., 2010). Maximum-likelihood analysis was performed using RAxML v. 8 (Stamatakis et al., 2005), specifying an LT model of amino acid replacement and a gamma model of rate heterogeneity $(+\mathrm{G})$ as selected by ProtTest v. 3.2 (Darriba et al., 2011). Nodal support was estimated with 500 bootstrap iterations.

\section{Gene Cloning}

All gene fragments were isolated by means of PCR with gene specific primers. Fragments were cloned into pCR-IITOPO or pCR4-TOPO vectors (Life Technologies). Sequences are available under accession numbers LN849082 (Ek-fz1), LN849083 (Ek-fz2), LN849084 (Ek-fz3), LN849085 (Ek$f z 4), \quad$ LN849078 (Gm-fz1), LN849079 (Gm-fz2), LN849080 (Gm-fz3), LN849081 (Gm-fz4). The Parasteatoda sequences are all available from the Assembled Searchable Giant Arthropod Read Database (ASGARD) (http://asgard.rc.fas. harvard.edu/) (Zeng and Extavour, 2012): Pt-fz1 (Locus 3605), Pt-fz2 (Locus 1), Pt-fz4-1 (Locus 7239), and Pt-fz4-2 (Locus 2608). 


\section{Embryo Collection, Fixation, and Staging}

Mature females of Glomeris were collected from February to May in the Reichswald forest (Nordrhein-Westfalen, Germany) near Kranenburg. They were kept in plastic boxes filled with decomposing beech leaves and moist soil at $\sim 20^{\circ} \mathrm{C}$. Under these conditions females lay eggs for several weeks. Pregnant females of Euperipatoides were sampled between August and October in the Kanangra Boyd National Park, NSW, Australia, and were then kept at $\sim 8^{\circ} \mathrm{C}$ in plastic containers filled with moist moss. Under these conditions they survive for at least several months when fed with small crickets once a week. The Parasteatoda culture at Oxford Brookes University was founded by spiders from a strain collected and maintained in Göttingen. Adults were kept separately in plastic vials at $25^{\circ} \mathrm{C}$ and fed with crickets. Pholcus spiders were obtained from the laboratory stock in Göttingen (Pechmann et al., 2011) and maintained like Parasteatoda, except that adults were fed with large flies like Musca. Embryos were collected, fixed and stored as described in Janssen et al. (2004) for Glomeris, in Janssen et al. (2015b) for Euperipatoides, and in Akiyama-Oda and Oda (2003) for Parasteatoda with slight modifications. Glomeris, Euperipatoides, and Parasteatoda embryos were staged according to Janssen et al. (2004); Janssen and Budd (2013), and Mittmann and Wolff (2012), respectively.

\section{Whole Mount In situ Hybridization and Nuclear Staining}

In situ hybridization of Euperipatoides and Glomeris embryos was performed as described in Janssen et al. (2015b) and Prpic and Tautz (2003), respectively. Embryos were hybridized with Digoxigenin-labeled RNA probes at $62^{\circ} \mathrm{C}$ for at least $16 \mathrm{~h}$. Parasteatoda in situ hybridization was carried out after the wholemount protocol for spiders (Prpic et al., 2008) with minor modifications. Primers used to generate probes are available on request.

Nuclear staining was performed by incubation in $1 \mu \mathrm{g} / \mathrm{ml} \mathrm{4-}$ 6-diamidin-2-phenylindol (DAPI) in phosphate-buffered saline with $0.1 \%$ Tween-20 for $30 \mathrm{~min}$.

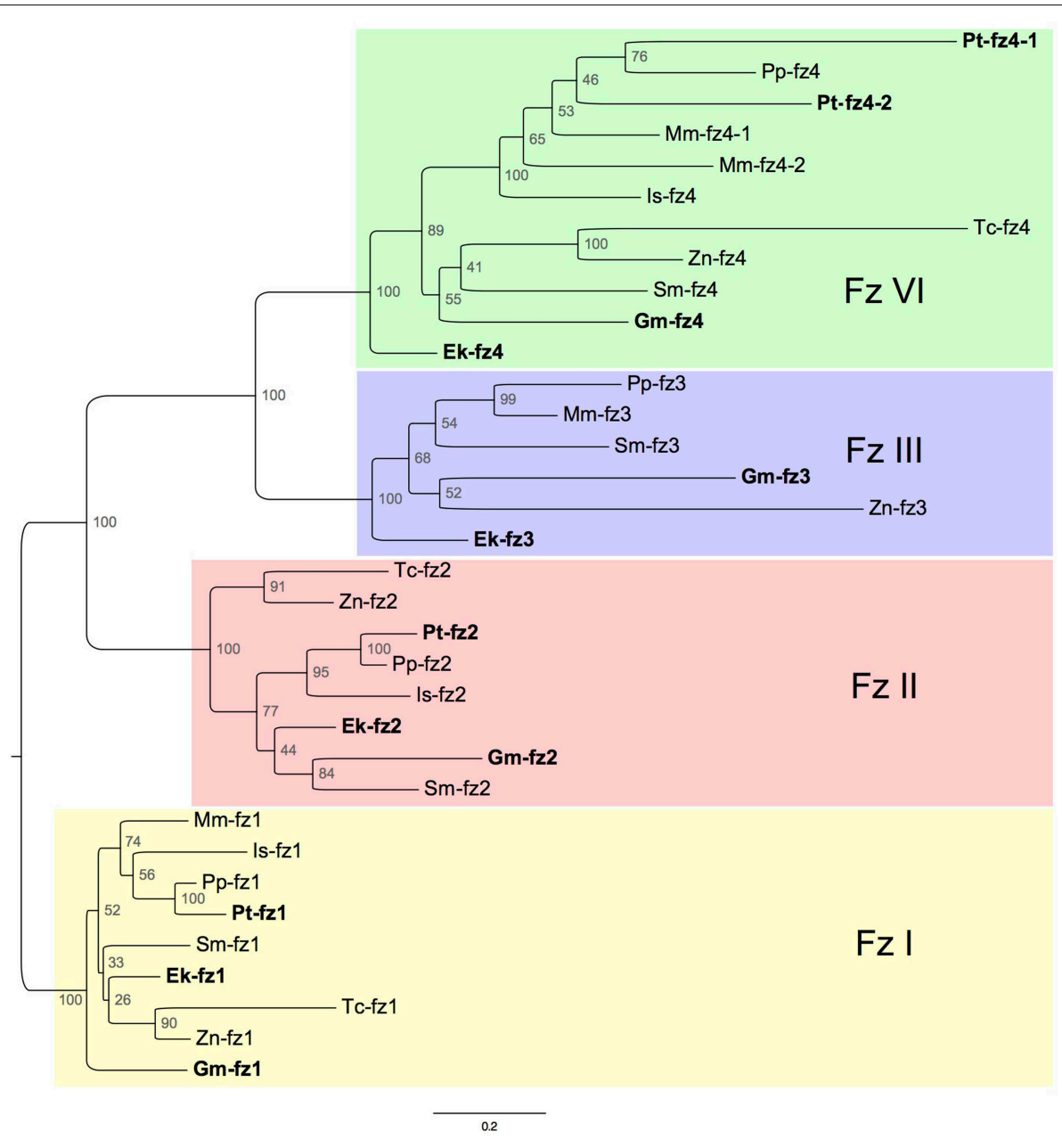

FIGURE 1 | Maximum-likelihood tree inferred from the analysis of conserved regions in frizzled genes of nine selected panarthropod species (Figure S1 shows our results from the same species while including Drosophila). Node values indicate bootstrap support. Ek,
Euperipatoides kanangrensis; Gm, Glomeris marginata; Pt, Parasteatoda tepidariorum; Mm, Mesobuthus martensii; Is, Ixodes scapularis: Tc, Tribolium castaneum; Zn, Zootermopsis nevadensis; Sm, Strigamia maritima; Pp, Pholcus phalangioides. 


\section{Data Documentation}

Embryos were analyzed under a Leica dissection microscope equipped with a Leica DC100 (Glomeris and Euperipatoides) or with a Jenoptik ProgRes C3 (Parasteatoda) digital camera. Linear corrections of brightness, contrast, and color values were performed with Adobe Photoshop.

\section{Results and Discussion}

\section{Phylogeny of the Panarthropod Frizzled Receptors}

Coding sequences of all putative $\mathrm{Fz}$ receptors recovered from genomic or transcriptomic resources for 10 panarthropod species were mostly complete. Exceptions include the Mm-fz1 sequence, which appears to be truncated after transmembrane domain 5. Gm-fz2, Zn-fz3, and Mm-fz4-1 sequences are possibly incomplete at the $\mathrm{N}$-terminus (Figure S1).

Phylogenetic reconstruction using maximum likelihood recovered four distinct orthology groups: FzI, FzII, FzIII, and FzIV (Figure S2). This is in concordance with previous findings in other metazoans (Schenkelaars et al., 2015). To avoid insect overrepresentation and potential disrupting effects of the derived Dm-fz3 and Dm-fz4 sequences, Drosophila sequences were omitted from our final analysis (Figure 1). This had little influence on the topology of FzI, II, and IV orthology groups, but led to clustering of the Glomeris Gm-fz3 sequence with the other non-onychophoran sequences within the FzIII orthology group.

In all investigated species, a single FzI ortholog was identified. FzII orthologs are present in the genomic resources of all species, except for the genome of the scorpion Mesobuthus. FzIV orthologs were also found in all investigated species. Moreover, it appears that a duplication of FzIV can either be traced to the base of the Arachnopulmonata (Scorpiones and Tetrapulmonata-including spiders) (Sharma et al., 2014), or happened independently in spiders and scorpions (Figures 1, 2). The only orthology group that showed gene loss in representatives of both the Chelicerata and Mandibulata was FzIII (Figures 1, 2).

\section{Fz Sequence Variation}

$\mathrm{Fz}$ receptors contain a conserved amino-terminal extracellular region that mediates binding to Wnt ligands: the cysteine-rich domain (CRD) (Figure 3 and Figure S1). The highest sequence similarity among panarthropod Fz CRD domains was found in the FzI orthology group (only nine sites with $<60 \%$ consensus), whereas the FzIII CRD sequences are most divergent (30 sites with $<60 \%$ consensus) (Figure 3). Moreover, the Drosophila Dm-fz1, Dm-fz3 and Dm-fz4 sequences stand out due to their relatively large number of deviations from the consensus of their respective orthology groups (Figure 3). This is consistent with long branches observed for these Drosophila sequences in our phylogenetic reconstruction (Figure S2), and is an indication that insights gained from functional studies of $\mathrm{Fz}$ receptors in Drosophila might not be representative of their function in other panarthropods.

A second functional domain is the KTxxxW motif, which is typically found directly after the seventh transmembrane

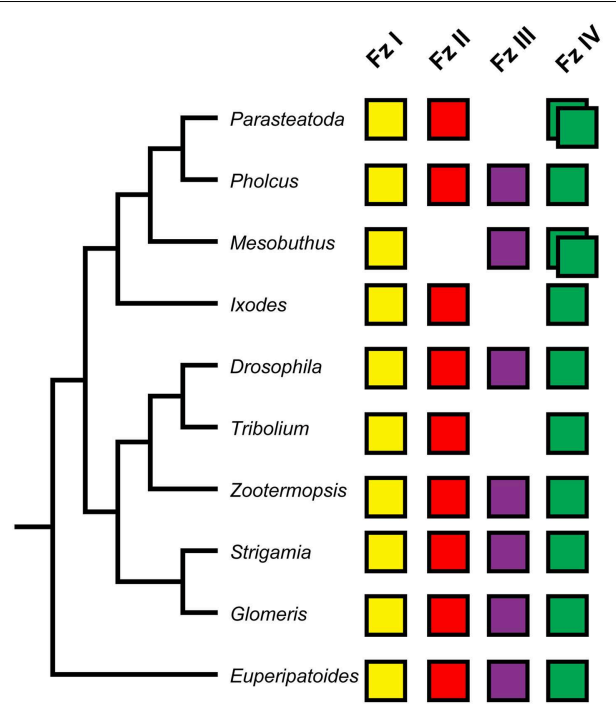

FIGURE 2 | Panarthropod frizzled genes. The four frizzled orthology groups are represented by colored boxes. The absence of a box indicates the loss of a particular frizzled ortholog. Duplicated frizzled genes are represented by two overlapping boxes. Phylogenetic relationships of the species included are indicated by the tree on the left.

domain (Huang and Klein, 2004). In Drosophila and vertebrates, this intracellular motif has been associated with binding to proteins of the Disheveled family. Alignment of the KTxxxW motif from panarthropods (Figure 3, positions 548-566) shows high sequence similarity in the FzI/II orthology groups, and less similarity for the FzIII/IV sequences. Interestingly, as with Drosophila Dm-fz3 and Dm-fz4, the tryptophan of this motif has been lost in both of the spider Parasteatoda Pt-fz4 paralogs, which could indicate a change or loss of the binding affinity of this motif in these two proteins. The tryptophan residue has, however, been retained in most other panarthropods FzIII/IV sequences, including the spider Pholcus (Figure 3 and Figure S1).

\section{Embryonic Expression of Fz1 Genes}

We identified orthologs of the FzI subfamily in Euperipatoides as well as the two arthropods, Glomeris, and Parasteatoda (Figures 1, 2).

In early embryonic stages of Euperipatoides, Ek-fz1 is expressed ubiquitously except for the posterior segment addition zone (SAZ), but is then upregulated in a segmental pattern in transverse stripes (Figure 4A). In the head lobes, expression is observed in a patch-like domain at the posterior margin of each of the two hemispheres (Figures 4A,B). Later, expression disappears from the tip of the outgrowing appendages (Figures 4C,D) and cells in the nervous system express $E k-f z 1$ at higher levels (Figure 4C). This expression profile persists through to later developmental stages (Figure 4E).

$G m-f z 1$ is expressed ubiquitously and uniformly at all investigated stages of embryogenesis in Glomeris (Figures 5A-D). In Parasteatoda, Pt-fz1 is also probably ubiquitously expressed at low levels, but the most prominent expression of $P t-f z 1$ expression starts at stage 9.1 in the 


\begin{tabular}{|c|c|}
\hline & 2 \\
\hline Fz I & RCEPITIPLCKDIQYNETIMPNLLNHQKQEDAGLEVHQFFPLVKVQCSPDLQFFLCSMYAPVCT-ILE*A \\
\hline Ek-fzl & RCEPITIPLCNDIQYNETIMPNLLNHQKQEDAGLEVHQFFPLVKVQCSPDLKEFLCAMYAPVCT-ILERA \\
\hline $\mathrm{Gm}-\mathrm{fz} 1$ & CEPISIPLCKDLQYNETIMPNLINHQKQEDAGLEVHQFFPLVKVQCSLELQFFLCAIYAPVCT-IIDRA \\
\hline Pt-fzl & RCEPITIPLCKDIPYNETIMPNLLNHQKQEDAGLEVHQFFPLVKVQCSPDLQFFLCTMYAPVCT-IIEKA \\
\hline $\mathrm{Dm}-\mathrm{fz}$ & RCEPITISICKNIPYNMTMPNLIGHTKEEAGLEVHQFAPLVKIGCSDDLQLFLCSLYVPVCT-ILERP \\
\hline Fz II & 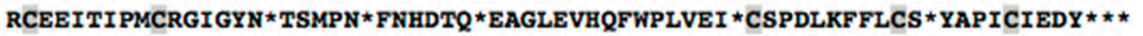 \\
\hline Ek-fz2 & KCEEITIPMCRGIGYNYTYMPNQFNHDSEEAGLEVHQFWPLVEIQCSPDLKFFLCSKYAPICIEDYHQP \\
\hline $\mathrm{Gm}-\mathrm{fz} 2$ & 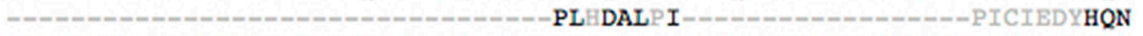 \\
\hline Pt-fz2 & RCEEITIPMCKGIGYNYTSMPNKFHHDQEEAGLEVHQFWPLVEIHCSDDLLFFLCSLYAPICIEDYHGQ \\
\hline $\mathrm{Dm}-\mathrm{fz} 2$ & RCEEITIPMCRGIGYNMTSFPEMNHETQDEAGLEVHQFWPLVEIKCSPDLKEFLCSMYTPICLEDYHKP \\
\hline Fz III & 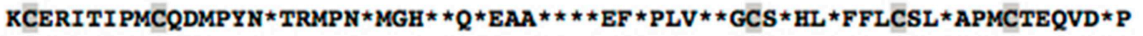 \\
\hline Ek-fz3 & KCERITIPMCADMPYNMTRMPNYNHD RDAALEVHEF FPLVKYGCSKHL KEFLCSLFAPMCTEQVDIP \\
\hline $\mathrm{Gm}-\mathrm{fz} 3$ & KCERISVPFCKDMPYNMTRLPNIVGQETMADASNSLL YQTLVRTGCSKHLTFFLCSLYTPMCSEQVDVA \\
\hline $\mathrm{Pp}-\mathrm{fz} 3$ & KCERITIPMCQDMPYNLTRMPNMGQTD SEAA IKVHEFFPLVEVGCSRHLKFFLCSIFAPMCTEQVDVP \\
\hline $\mathrm{Dm}-\mathrm{fz} 3$ & QCQPIAVSACQ GLGYNMALPLAGHTN LEAELQIAKLVPLIESGCSRRARELLCSSLFPLCTPDVPR \\
\hline Fz IV & *CEPIRIEMC * *LGYNVTGMPNLVGHELQQDA * *QLQTF*PLIQYGCSSQL *FFLCSVYVPMCTEKV * $P$ \\
\hline Ek-fz 4 & TCDPIKIEMCKGLGYNVTGMPNLVGHELQQDADMQLQTETPLIQYGCSSQLKFFLCSVYVPMCTEKVSFP \\
\hline $\mathrm{Gm}-\mathrm{fz} 4$ & --PPTRR---SGLGYNVTGMPNLVGHELQQDADLLLQTETPLIQYGCSSQLRFFLCSVYVPMCTEKVALP \\
\hline Pt-Fz4-1 & TCEPIRIEI CRDI GYNVTGMPNLVGHELQQDAQLQLQTEQPLIQYGCSSR QFFLCSVYVPMCTEKVPDP \\
\hline Pt-Fz4-2 & TCEPIRIDSCKNIGYNVTGMPNF GHDM $O H D A L I Q T F M P L Q Y G C S S R$ SFFLCSVYVPMCTDKVPQP \\
\hline $\mathrm{Dm}-\mathrm{fz} 4$ & QCETIRIEMCRKIGYNETSMPNLVGNEM TDVEYTLQTEAPLIEYDCSSQLKLFLCAAYVPMCTPKAPVH \\
\hline
\end{tabular}

\begin{tabular}{|c|c|c|}
\hline & 167 & 548 \\
\hline Fz I & 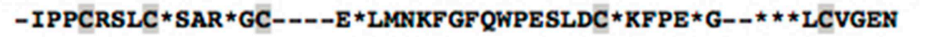 & SG WIWSGKT $-\mathbf{F}$ *SWK-NFY * \\
\hline $\mathrm{Ek}-\mathrm{fz} 1$ & -IPPCKSLCMSAKNGC----ESLMNKF GFQWPESLDCNQFP-EA--NDDICVGQN & SGFWIWSGKT-FSSWK-NFYC \\
\hline $\mathrm{Gm}-\mathrm{fz} 1$ & -LPPCRNLCLSARNGC----EELMNKF GF QWPDSLDCSKFPESG--GPDLCVGEN & SGGWIWSWKT-VASWK-KFYE \\
\hline Pt-fzl & -IPPCRSLCLKAREGC----ERLMNNFGQWPKSLDCDKFPEAG--EEALCVGEN & SGVWIWSGKT-FTTWK-NVYL \\
\hline$m-f z$ & -IPPCRSLCESARV-C----EKLMKTYNENWPENLECSKFPVHG--GEDLCVAEN & SSVWLYSSKT-MVSWR-NFVE \\
\hline Fz II & -LPACRSVCERARAGC- $--A P I M \star Q Y G F \star W P E R M N C \star \star L P \star Y G A D \star$ & ४SGK: \\
\hline $\mathrm{Ek}-\mathrm{fz} 2$ & -LPACRSVCERARAGC----APL GQYGF PWPERMNCEALPYG-DDKQLCMDSK & WSGKT-LDSh \\
\hline $\mathrm{Gm}-\mathrm{fz} 2$ & -LPACRSVCERAKAGC----SPLMRYGFAWPERMNCDRLPTYG-DPNQLCMDSK & SGLWVLSGKT-IDSWR-RFKI \\
\hline Pt-fz2 & -LPACYSVCERAKAGC----APIMHKYGFVWPERMNCDDFPRYG-DQEHLCMDAK & SGCWITSTKT-IESWRNRLCS \\
\hline$m-f z 2$ & -LPVCRSVCERARSGC----APIMQQYSE EWPERMACEHLPLHG-DPDNLCMEQP & SGVWIWSGKT-LESWR-RFWR \\
\hline Fz III & 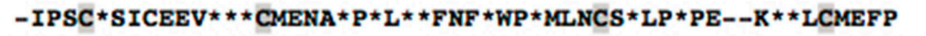 & 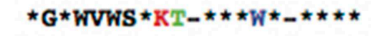 \\
\hline Ek-fz3 & -IPSCKSICDEVKSKC----LPL KQFNE D WPLINCSRLPIPE---NGLCMEFP & SGMNIWSTKT-WYS \\
\hline $\mathrm{Gm}-\mathrm{fz} 3$ & -IPSCRSICEEVRSRC----EPFLQGEHIPWPRALNCSQLPVHS--KDELCMEFP & SGMWVWS-KNTLEAV \\
\hline $\mathrm{Pp}-\mathrm{fz} 3$ & $-I P S C Q S I C E E V K S K C----S P V L Q S E H E N W P$ PMLDCSKLPVPE--KNGLCMEYP & TGVWVWSSKT-WNSWG-KFFS \\
\hline $\mathrm{Dm}-\mathrm{fz} 3$ & -VAACKLLCETVRGECMENAPPELMEL---WPSFLNCDGLPQPE--KHELCMQIP & TGLWVWSRKT-CESYRNRLGA \\
\hline Fz IV & $A I G P C R * L C E \star V R \star R C----Q P V L Q E F G F P W P \star A L N C S K F P P E N-N *$ HMCMEGP & 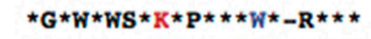 \\
\hline Ek-fz 4 & -IGPCRPLCENVKNRC----EPVLQEFGFPWPSALNCSKFPPEN-NQHDMCMDGP & SGMWIWSTKT-LTSWQ-RFT \\
\hline $\mathrm{Gm}-\mathrm{fz} 4$ & -IGPCRPLCETVRSRC----QPILQEFGFPWPSALNCSKFPPVN-NQKHMCMDGP & SAM VWSSKT-LDSWR-RAWR \\
\hline Pt-Fz4-1 & -IGPCRSLCEDIRDKC----QPVLQEFGFPWPSGNCSKFPPQN-NNKHMCMEGP & ASFVIFTSSARKRAFK-TLCR \\
\hline Pt-Fz4- & -IGPCRSLCESVQERC----EPVILEFGEQWPVALNCSQFPPVN-NEKHMCMEGP & TGVWLYTSRSP QLRRP-KLS \\
\hline $\mathrm{Dm}-\mathrm{fz} 4$ & AIGPCRSLCESVRIRC----HPVL, GFGFPWP PAIDCDKFPREN-NHETMCMEGP & CFAWVLGSI-STLYK-RQV \\
\hline
\end{tabular}

FIGURE 3 | Sequence alignments of the cystein rich domain (CRD) and KTxxxW motif of arthropod Frizzled proteins. Sixty percent consensus sequences, based on the alignment of the Frizzleds from all 10 species depicted in Figure 2, are shown in bold type separately for orthology groups Fzl-IV. Individual sequences are shown for Euperipatoides kanangrensis (Ek), Glomeris marginata (Gk), Parasteatoda tepidariorum (Pt), and Drosophila melanogaster (Dm).
Chelicerate $\mathrm{fz} 3$, for which no Parasteatoda sequence was found, is represented by the Pholcus phalangioides sequence (Pp-fz3). Cysteine residues are shaded gray in $\mathrm{CRD}$ consensus sequences. Black font indicates deviation from the consensus sequence. Red, green, and blue fonts are used to highlight the Lysine, Threonine, and Tryptophan residues in the $\mathrm{KT} x \mathrm{xxW}$ motif. Amino acid positions are indicated according to Dm-fz. ventral neuroectoderm (Figure 6), which then persists through subsequent stages. However, no specific spatial expression of $P t-f z 1$ was detected before or after this stage of embryogenesis in Parasteatoda.

In the early embryos of both Drosophila and Tribolium, the $f z 1$ genes are initially uniformly expressed (Park et al., 1994; Müller et al., 1999; Beermann et al., 2011). While this ubiquitous expression persists in Tribolium embryos (Beermann et al., 2011), the expression of Drosophila Fz resolves into broad stripes between the Engrailed (En) expressing cells (Müller et al., 1999). In both of these insects, Fz appears to function together with the respective $\mathrm{Fz} 2$ receptor to regulate segment formation via the Wingless (Wg) ligand (Chen and Struhl, 1999; Müller et al., 1999; Bolognesi et al., 2008; Beermann et al., 2011). The expression of $f z 1$ genes in Euperipatoides, Glomeris and Parasteatoda is consistent with a role for $f z 1$ genes in segmentation across the panarthropods. 


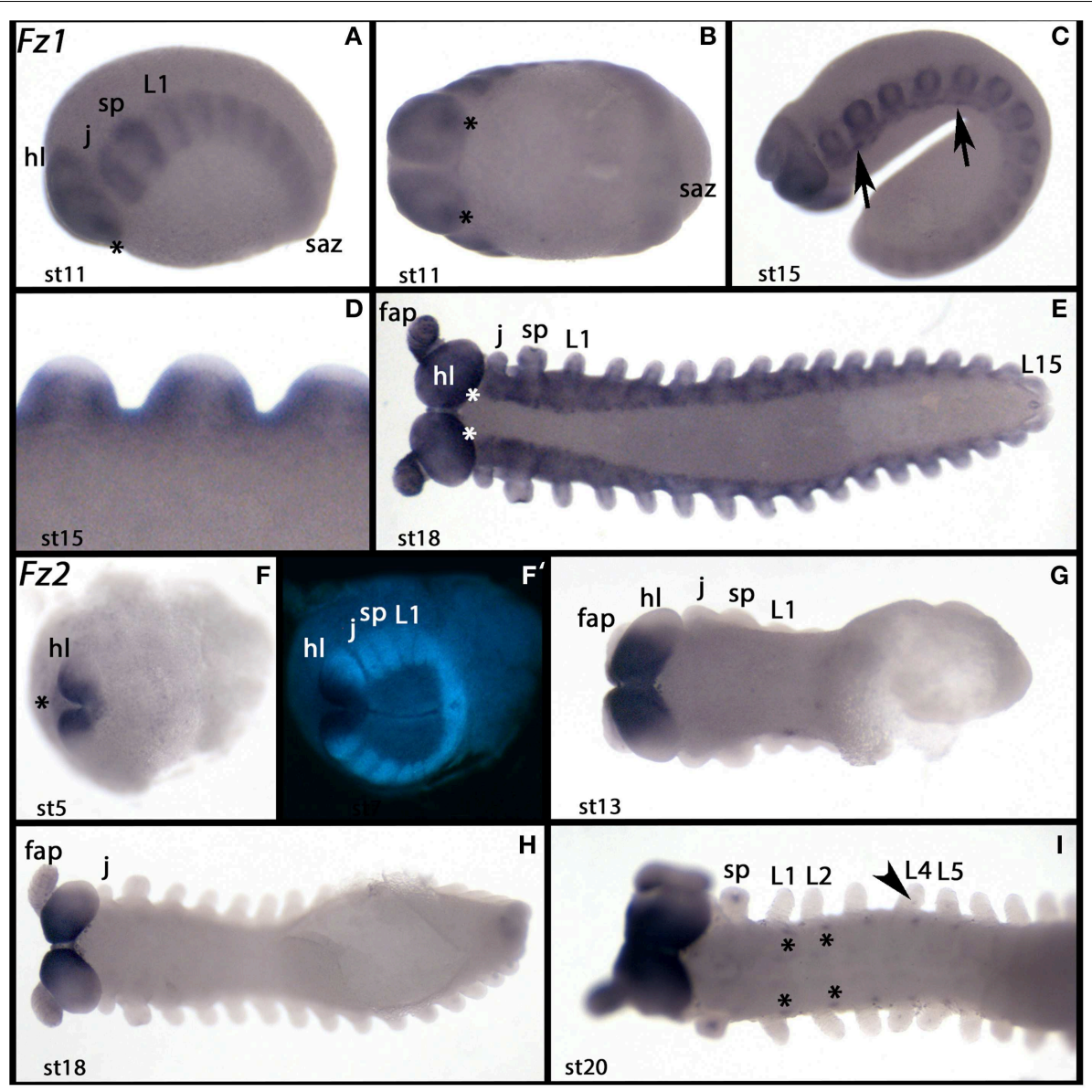

FIGURE 4 | Expression of Euperipatoides $\mathbf{f z} \mathbf{1}$ and $\mathbf{f z 2}$. In all panels anterior is to the left. (A-E) Show expression of Ek-fz1. (F-I) Show expression of Ek-fz2. (A,C) Lateral views. (B,E-I) Ventral views. (D) Dorsal view. ( $\left.\mathbf{F}^{\prime}\right)$ Shows the DAPI counterstaining of the embryo shown in (F) (same position). Developmental stages are indicated. Asterisks in $\mathbf{( A , B , E ) ~ m a r k ~ e x p r e s s i o n ~ a t ~}$ the posterior rim of the head lobes. Arrows in (C) point to clusters of cells in the ventral nervous system with upregulated expression. The asterisk in (F) marks expression in extraembryonic tissue. Asterisks in (I) mark expression in the ventral nervous system at the base of the walking limbs. The arrow in (I) points to dot-like expression in the walking limb L4. Note that this expression is restricted to L4 and L5. fap, frontal appendage; hl, head lobe; j, jaw; L, walking limb; saz, segment addition zone; sp, slime papilla; st, stage.
Drosophila $f z$ is also expressed in other tissues including the imaginal discs and central nervous system (Park et al., 1994). However, $f z$ mutants can produce viable adult flies that only exhibit defects in planar cell polarity (reviewed in Strutt, 2003). This suggests that, like during segmentation (see above), this receptor functions in combination with other $\mathrm{Fz}$ proteins in a range of roles in Drosophila, and given its widely distributed expression in Euperipatoides, Glomeris, and Parasteatoda, this is probably the case in other panarthropods.

\section{Embryonic Expression of Fz2 Genes}

We identified orthologs of the FzII subfamily in all three of the panarthropods that we investigated (Figures 1, 2).

In Euperipatoides, $E k-f z 2$ is strongly expressed during embryogenesis in the ventral region of the head lobes, including the mouth that forms from the anterior of the mouth-anus furrow (Janssen et al., 2015a). Interestingly, tissue anterior to the head lobes proper also expresses $E k-f z 2$ (Figures $4 \mathbf{F}, \mathbf{F}^{\prime}$ ). This remains the only detectable expression (Figures $4 \mathbf{G}, \mathbf{H}$ ) until stage 20 when dot-like domains appear in an anterior to posterior order at the base of the walking limbs, and in a small domain ventrally at the base of the third and fourth walking limb (Figure 4I). The latter expression may be correlated with the development of the nephridia, which are more pronounced in these two segments (Mayer, 2006).

In Glomeris, $G m-f z 2$ is expressed in an anterior domain covering $\sim 30 \%$ of the early post-blastoderm stage embryo (Figure 5E). At later stages this domain includes the anterior and median region of the developing head lobes including the stomodaeum (Figures S3A-C). At approximately stage 0.5, a faint transverse stripe of expression appears (Figure S3A). This stripe likely corresponds to expression in the mandibular segment as is obvious in later stages (cf. Figures S3A,B). Within the mandibular segment, expression is restricted to the anterior of the developing mandibles (Figure 5F and Figures S3B,C). The dorsal segmental tissue that forms at stage 3 (Janssen et al., 2004) expresses $G m-f z 2$ in a complex pattern (Figures $\mathbf{5 F}-\mathbf{H}$ and 


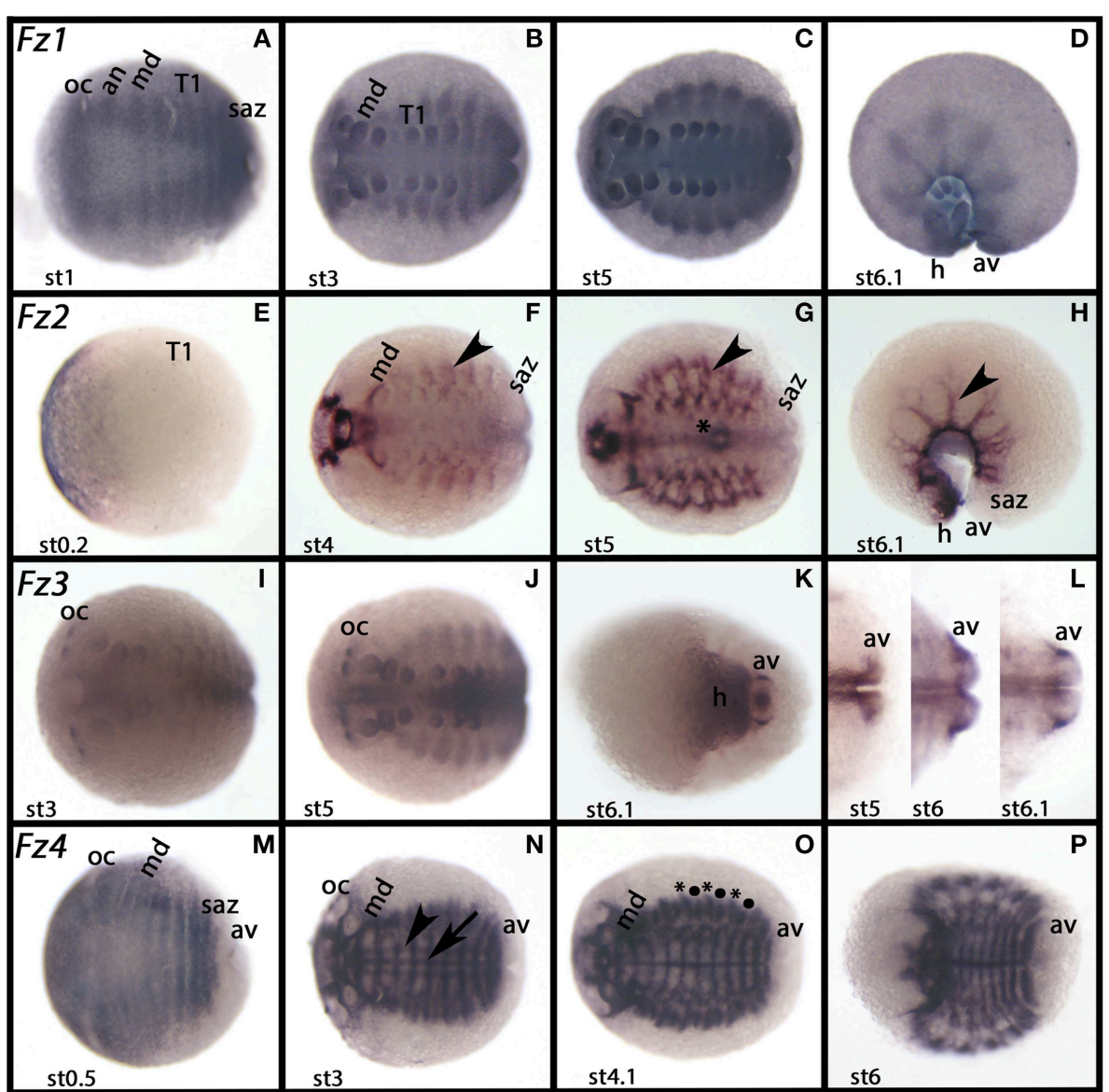

FIGURE 5 | Expression of Glomeris frizzled genes. In all panels anterior is to the left. Ventral views, except for (D) and (H) (lateral views). Developmental stages are indicated. (A-D) Ubiquitous expression of $G m-f z 1$. (E-H) Expression of $G m-f z 2$, (I-L) Expression of $G m-f z 3$. (M-P) Expression of $G m-f z 4$. The arrow in (N) points to the midline; the arrowhead points to expression parallel to the midline.
Asterisks and filled circles $(\bullet)$ in (0) mark two domains of expression in every dorsal segmental unit of the trunk. The arrowhead in (F-H) marks dorsal expression. The asterisk in (G) marks expression in the midgut. an, antennal segment; av, anal valve; h, head; md, mandibular segment; oc, ocular region; saz, segment addition zone, st, stage; T, trunk segment.
Figure S3C), and $G m-f z 2$ is also expressed in the midgut from stage 5 onwards (Figure 5G).

In Parasteatoda, Pt-fz2 expression commences at stage 5 in a broad ring around the germ disc (Figure 6B). After radial symmetry is broken (Akiyama-Oda and Oda, 2003), Pt-fz2 continues to be expressed in an anterior stripe (Figure 6C), which widens at a later stage, but the expression intensity remains comparable. At stage $8.1 \mathrm{Pt}-\mathrm{fz} 2$ appears to be expressed at the anterior border of the germ band and in the forming L1L4 segments, although the expression in the L2-L4 segments is much broader than in L1 (Figure 6D). At stage 8.2, when prosomal segmentation has become morphologically visible and the first opisthosomal segment (O1) has formed, $P t-f z 2$ is expressed in the anterior compartment of each segment and in the segmental grooves (see Figure 6E). There is also a narrow Pt$f z 2$ expression domain at the anterior margin of the germ band (see Figure 6E). However, no expression is observed in the SAZ. Subsequently, Pt-fz2 expression in each segment contracts to the segmental grooves, and the anterior-most band expands to a wedge shaped domain in the anterior precheliceral lobes, partially encompassing the stomodaeum (Figure 6F). Strong expression is also observed in the ventral neuroectoderm and the dorsal periphery of each segment (see Figure 6F).

In Drosophila embryos, Dfz2 is also initially expressed in a broad domain but is absent from the anterior and posterior poles (Bhanot et al., 1996). Dfz2 expression then develops into broad segmental stripes posterior to En expressing cells, but unlike $\mathrm{Fz}$, this expression does not quite extend as far as the $w g$ expressing cells (Bhanot et al., 1996; Müller et al., 1999).

In Tribolium, like Drosophila, Glomeris, and Parasteatoda, Tc$f z 2$ is also initially expressed in a broad presegmental region that resolves into segmental stripes anterior to $w g$ expression (with the exception of the more complex pattern of $G m-f z 2$ ), and like in Parasteatoda and Glomeris, expression of Tc-fz2 in Tribolium is absent from the SAZ (Beermann et al., 2011).

Taken together, comparison of the expression of $f z 2$ genes across arthropods suggests that this member of the Fz family was employed in segmentation in the common ancestor of 


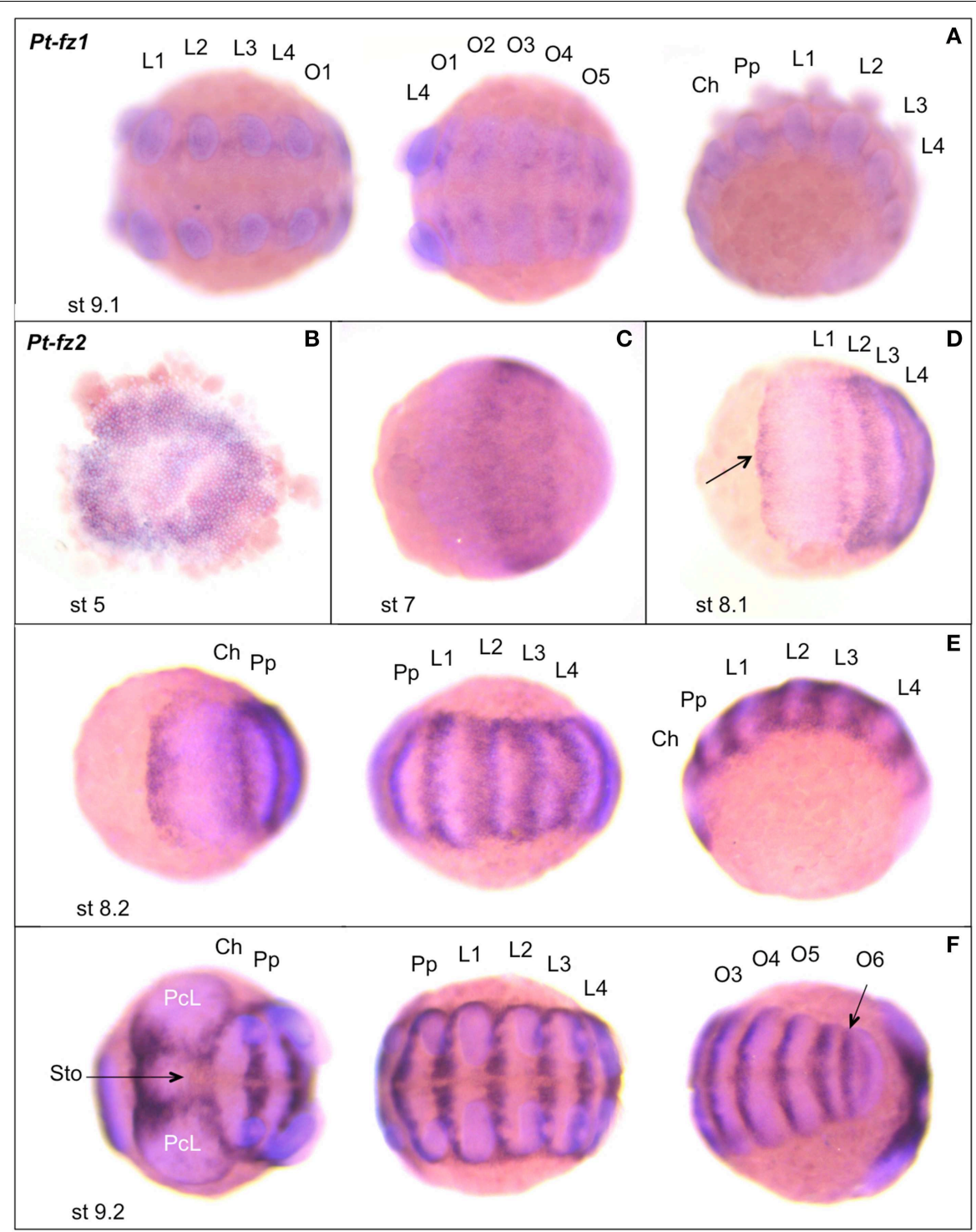

FIGURE 6 | Expression of Parasteatoda $\mathbf{f z} \mathbf{1}$ and $\mathbf{f z 2}$. In all panels anterior is to the left. (A) Pt-fz1 expression, from left to right, in a ventral view of the posterior prosomal germ band, a ventral view of the opisthosomal germ band and a lateral view. (B-E) Pt-fz2 expression. (B) Flatmounted germ disc. (C,D) Early germ band (anterior prosoma). (E) Germ band (anterior prosoma, posterior prosoma, and lateral view). (F)
Germ band (anterior prosoma, posterior prosoma, and opisthosoma/SAZ view). The arrow in (D) indicates the expression domain along the anterior border of the germ band. The arrow in (F) marks expression at the stomodaeum. Developmental stages are indicated. L, walking limb; O, opisthosomal segment; Ch, cheliceres; Pp, pedipalps; PcL, precheliceral lobe; Sto, stomodaeum; st, stage. these animals. In developing insect segments it is most likely that Fz2 is bound by Wg, however, in Parasteatoda the most probable candidates are Wnt5, Wnt8, and Wnt16 because $w g$ is not expressed in an obvious segmental pattern in this spider (Janssen et al., 2010). Moreover, given the lack of a segmental pattern of expression of $f z 2$ in Euperipatoides (Figure 4), it remains unclear if this gene played a similar role in the ancestor of panarthropods. It is also evident from our results that the anterior expression of $f z 2$ in the median head region is conserved in Glomeris, Euperipatoides, Tribolium, and Parasteatoda and therefore probably represents a conserved ancestral expression domain of this gene.

\section{Embryonic Expression of fz3 Genes}

We identified orthologs of the FzIII subfamily in both Euperipatoides and Glomeris, but like Tribolium, Parasteatoda lacks a $f z 3$ gene (Figures 1,2).

$E k-f z 3$ is expressed segmentally in the form of transverse stripes (Figures 7A,C). These stripes are level with the position of the limb primordia (Figure 7A). The tips of outgrowing limbs, however, are free from expression (Figures 7D,E). Transcription in the head lobes is restricted to lateral tissue (Figures 7A,B,E) and complements that of $E k-f z 2$. Expression in the trunk segments complements that of $E k-f z 4$ (see below). The SAZ does not express $E k-f z 3$, but a faint signal is visible in the anus 


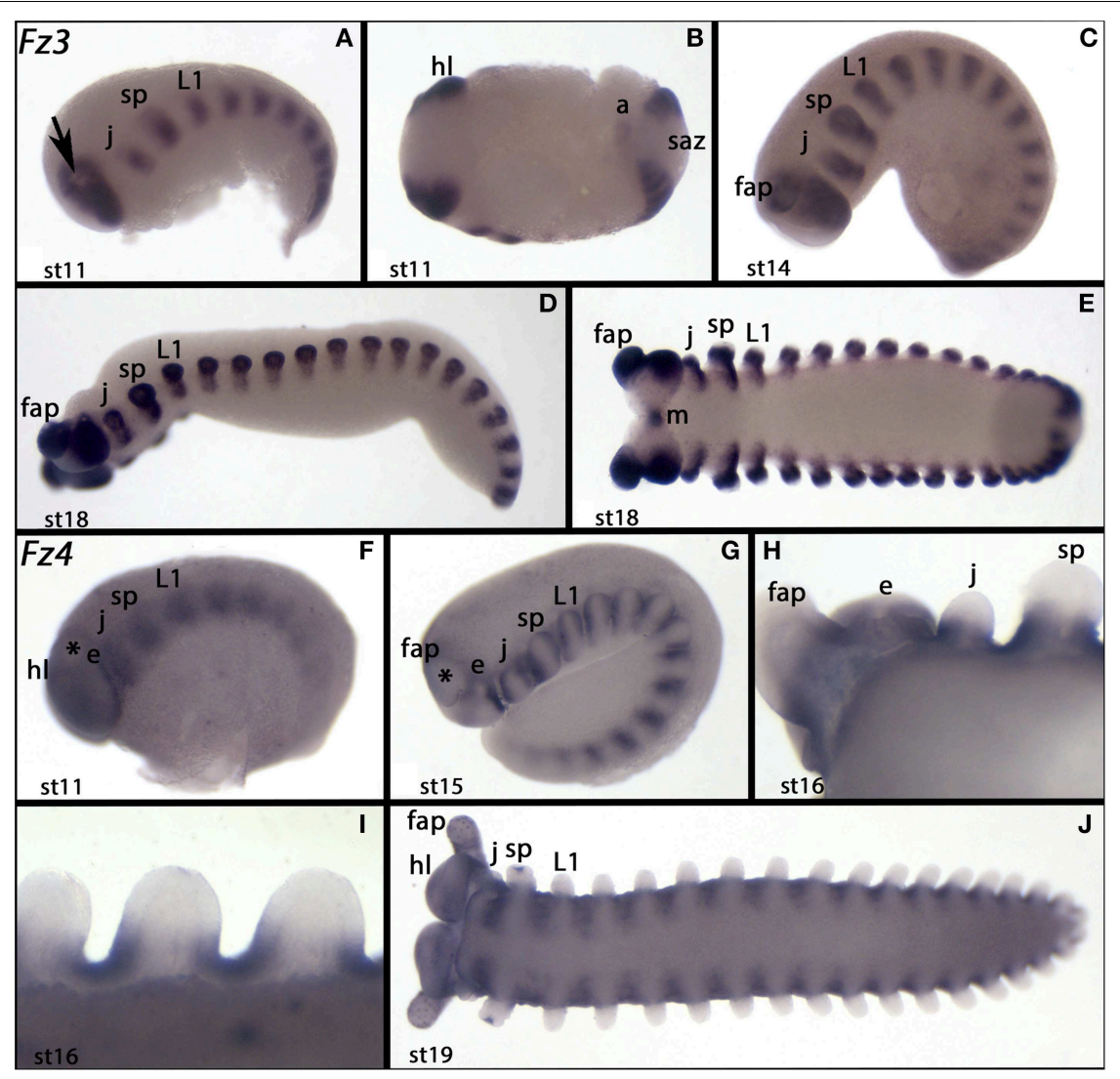

FIGURE 7 | Expression of Euperipatoides $f z 3$ and $f z 4$. In all panels anterior is to the left. (A-E) Show expression of Ek-fz3. (F-J) Show expression of $E k-f z 4$. (B,E,I,J) Ventral views. (A,C,D,F,G) Lateral views. (H)
Dorsal view. Developmental stages are indicated. The arrow in (A) and the asterisks in $\mathbf{( F )}$ and $(\mathbf{G})$ mark the primordium of the frontal appendages. Abbreviations as in Figure $\mathbf{4}$ and a, anus; e, eye; m, mouth.
(Figures 7D,E). At later developmental stages, expression is seen in the mouth (Figure 7E).

In Glomeris we did not detect any specific staining for $f z 3$ in embryos younger than stage 3 . From this stage onwards, $G m-f z 3$ is expressed in the form of two dots in each eye field (Figures 5I,J). At later developmental stages, expression appears in the lateral region of the anal valves (Figures 5K,L). Detection of $G m-f z 3$ transcripts requires prolonged staining time, therefore, it is unclear whether this gene is expressed ubiquitously at a low level, or if this weak signal represents background staining. It is possible that $G m-f z 3$ is also expressed in the developing gut (Figure 5).

The expression of $f z 3$ has been investigated previously in Drosophila, but it is not completely straightforward to relate these patterns to those we have observed in Glomeris and Euperipatoides. Drosophila $f z 3$ is expressed in segmentally reiterated stripes during later embryogenesis like that observed in Euperipatoides, as well as, for example, in the brain, eye and leg discs, and anal tissue like Glomeris and/or Euperipatoides (Sato et al., 1999; Sivasankaran et al., 2000). This may indicate that $f z 3$ played an ancestral role in tissues like the nervous system, eye, and appendage development, however, a survey of a wider range of panarthropod species that still possess a member of the FzIII subfamily is required to address this more fully.

\section{Embryonic Expression of Fz4 Genes}

We identified single copies of FzIV subfamily genes in Euperipatoides and Glomeris, and two $f z 4$ paralogs in Parasteatoda (Figures 1, 2).

The early expression of $E k-f z 4$ is comparable to that of $E k-f z 1 . E k-f z 4$ is expressed in a segmental fashion. Within the head lobes, the primordia of the frontal appendages, however, are free from expression as are the eyes and a wedge-shaped domain ventral to the eyes (Figure 7F). At later stages it becomes clear that segmental expression is initially located between the outgrowing limbs (Figure 7G). Expression in the limb buds is restricted to anterior and posterior proximal tissue (Figures 7G-I). Tissue ventral to the base of the limbs no longer expresses $E k-f z 4$ at these later stages, or only weakly (cf. Figures 7H,I). As the ventral nervous system grows out in the process of ventral closure, tissue ventral to the bases of the limbs remains free of transcripts (Figure 7J).

At early stages, Glomeris $G m-f z 4$ is expressed in all tissues except for a distinct region in the anterior head, possibly the eye-field (Figure 5M). Expression is then upregulated in segmental stripes in the SAZ and newly formed segments, but the anal valves remain free of expression throughout development (Figures 5M-P). 
Interestingly, $G m-f z 4$ is also expressed in the dorsal extraembryonic tissue in form of an anterior cap (Figures S3H-J). At stage 3, the anterior of the head lobes are free of expression. With the exception of the labrum, there is no detectable expression in the distal part of all appendages (Figures S3D-G). At all later stages, $G m-f z 4$ is strongly expressed in the midline (Figures $\mathbf{5 N}-\mathbf{P}$ ), but only at around stage 3 do longitudinal stripes of expression appear on either side of the midline (Figure 5N). In dorsal tissue, two domains per segmental unit exhibit recognizably higher expression (Figures 5N-P).

In Parasteatoda, $P t-f z 4-1$ expression commences at stage 8.2 in a stripe at the posterior of the $\mathrm{O} 2$ segment and in a stripe at the anterior portion of the SAZ (see Figure 8A). Faint $P t-f z 4-1$ expression can also be detected in the mesoderm of the forming limbs and in a distinct domain on each of the precheliceral lobes, at stage 9.1 (Figure 8B). Subsequently, $P t$ - $f z 4-1$ expression becomes stronger and more broadly expressed in the limb mesoderm and expands also in the ventral neuroectoderm (see Figure 8C). The expression in the precheliceral lobes continues during stage 9.2 (see Figure 8C). Then during stage 12, Pt-fz4-1 is strongly expressed in the limb mesoderm, in the labrum and in the mesoderm of the opisthosomal segments (see Figure 8D). As the brain differentiates, expression refines to the anterior border of the precheliceral lobes: the anlage of the prosomal shield that overgrows the brain in the following stages (Wolff and Hilbrant, 2011; Mittmann and Wolff, 2012) (Figure 8D).

Pt-fz4-2 expression arises in an anterior stripe at stage 6 (Figure 9A). Compared to the anterior $P t-f z 2$ stripe at a similar stage (see Figure 6C), this $P t-f z 4-2$ expression domain is narrower (Figures 9A,B). Later, at stage 8.2, Pt-fz4-2 is expressed in the segmental grooves in the prosomal and opisthosomal segments and in a ring around the future labrum (Figure 9C). The expression of $P t-f z 4-2$ retracts to the dorsal periphery of each segment at stage 9.2 and the domain around the labrum becomes more defined (Figure 9D). Pt-fz4-2 is also strongly expressed in the limb and opisthosomal mesoderm at stage 12 (Figure 9E). Anteriorly, Pt-fz4-2 expression continues in the labrum, and also appears in a domain directly anterior to it (Figure 9E).

The two $f z 4$ paralogs in Parasteatoda exhibit both similarities and differences in their expression. For example, both are expressed in the labrum and in similar patterns in the extended walking legs, but $f z 4-1$ is expressed earlier in the nervous system

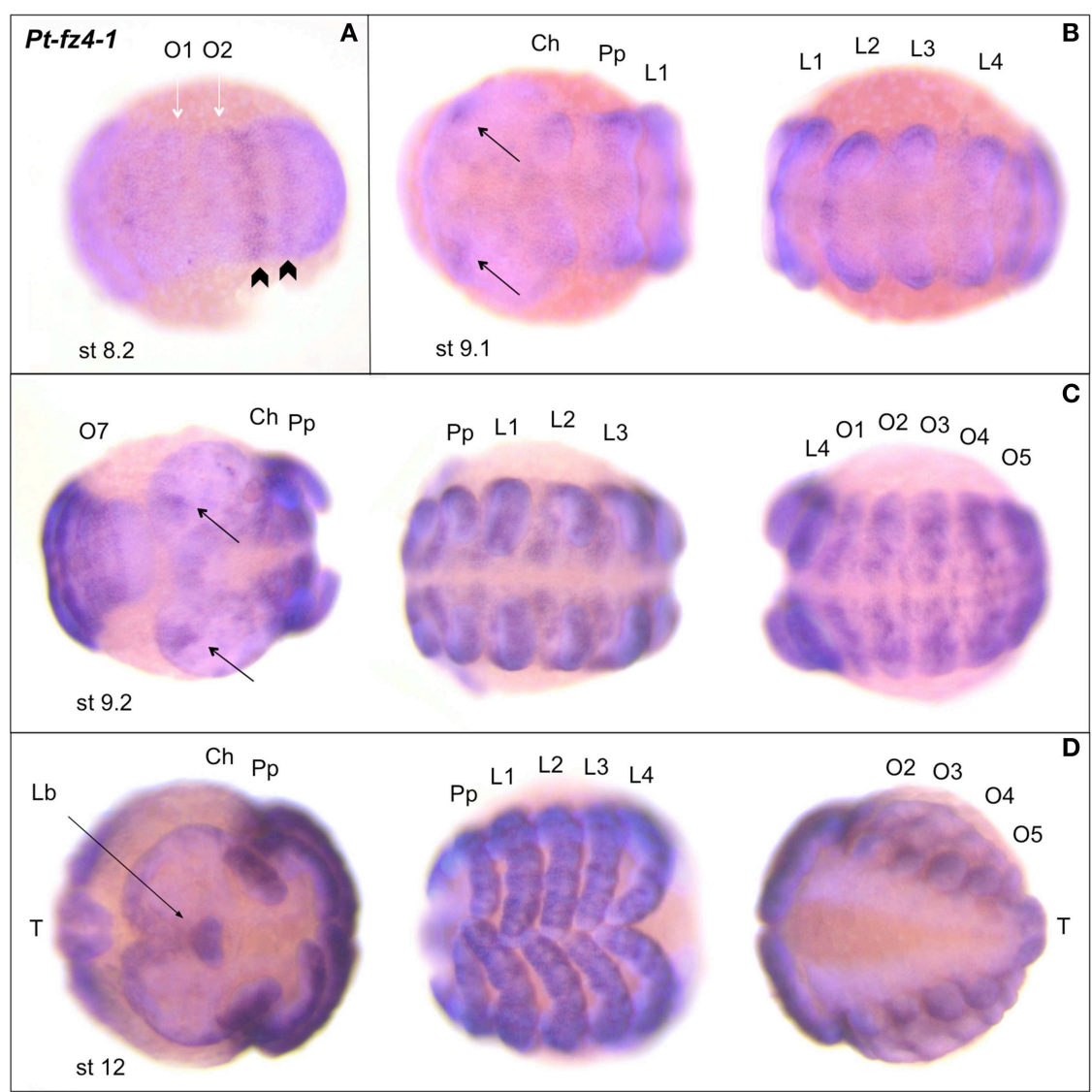

FIGURE 8 | Expression of Parasteatoda fz4-1. In all panels anterior is to the left. (A) Germ band (ventral opisthosomal/SAZ view). (B) Germ band (anterior (left) and posterior (right) prosoma). (C) Germ band (from left to right: posterior opisthosoma/SAZ and anterior prosoma, posterior prosoma and anterior opisthosoma). (D) Retracting germ band (anterior prosoma, posterior prosoma, opisthosoma). The white arrows in (A) indicate the $\mathrm{O} 1$ and $\mathrm{O} 2$ segments; the black arrowheads mark $P t-f z 4-1$ expression in $\mathrm{O} 2$ and the SAZ. Developmental stages are indicated. Abbreviations as in Figure 6. Lb, labrum; T, telson. 


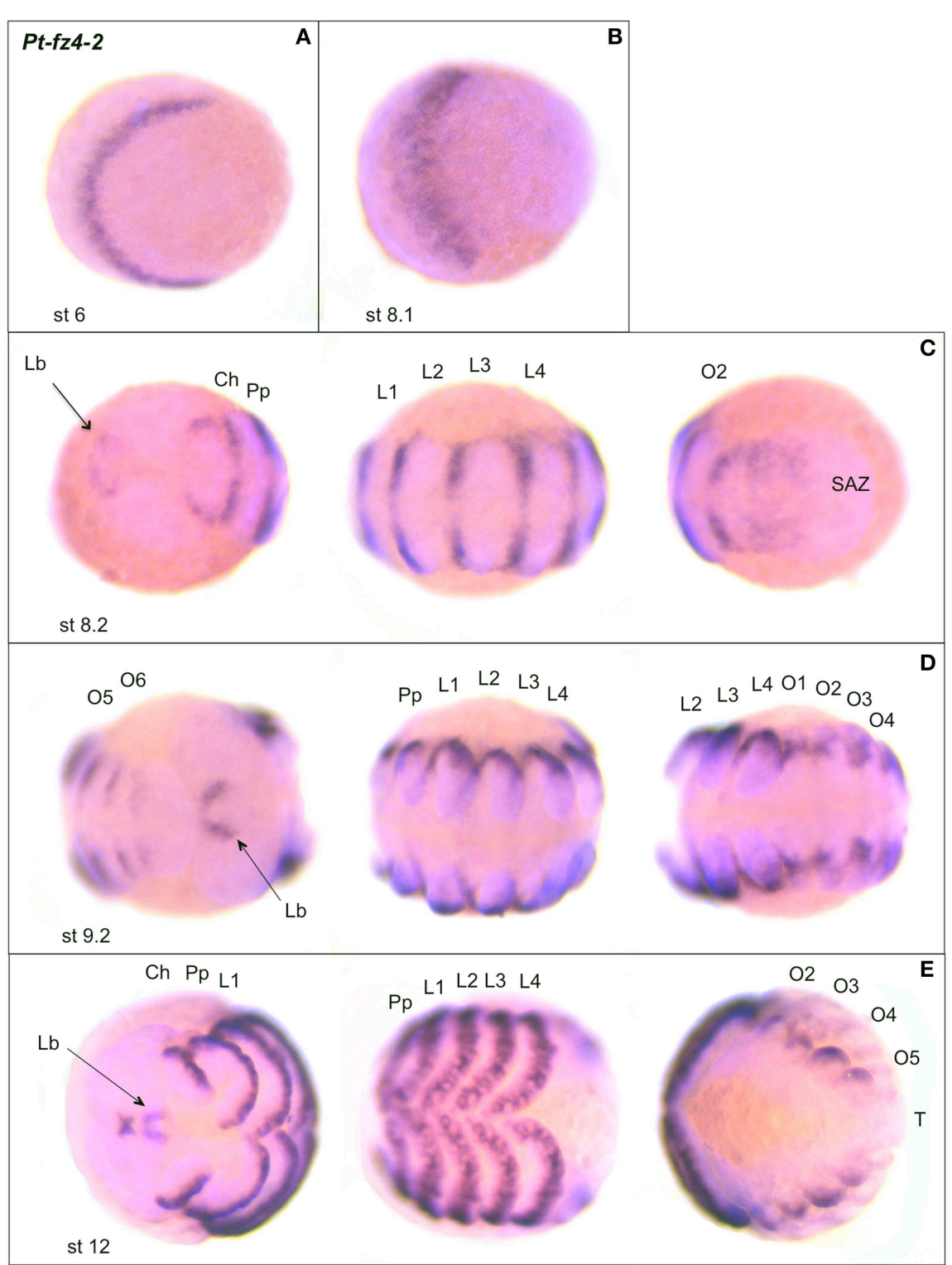

FIGURE 9 | Expression of Parasteatoda $\mathbf{f z 4 - 2 . ~ I n ~ a l l ~ p a n e l s ~ a n t e r i o r ~ i s ~ t o ~}$ the left. (A,B) Early germ band (anterior prosoma). (C) Germ band (from left to right: anterior prosoma, posterior prosoma and opisthosoma/SAZ). (D) Germ band (posterior opisthosoma/SAZ and anterior prosoma, posterior prosoma and anterior opisthosoma) (E) Retracting germ band (anterior prosoma, posterior prosoma, and opisthosoma). The arrows in (C-E) indicate expression in the labrum. Developmental stages are indicated. SAZ, segment addition zone, further abbreviations as in Figure 6. and head lobes, while $f z 4-2$ is expressed segmentally including in anterior segments (Figures 8, 9). Moreover, $f z 4-2$ expression appears in restricted domains at the segmental borders and the forming limbs compared to $f z 4-1$, which is more broadly expressed within the segments, limbs and the developing nervous system. These results may suggest that these two paralogs have been subject to subfunctionalization (Force et al., 1999; Lynch and Force, 2000).

It is clear from our comparative analysis and from previous work in Drosophila and Tribolium (Janson et al., 2001; Beermann et al., 2011) that genes of the FzIV family likely play a diverse range of roles during the development of panarthropods.
However, comparable patterns emerge among the arthropods, which suggest that these genes were involved in nervous system development, and possibly segment formation, as well as perhaps limb development more widely among panarthropods.

In Tribolium, $T c-f z 4$ appears to function together with $T c-f z 1$ to regulate leg development most likely by binding $\mathrm{Wg}$, although other Wnt ligands could be involved (Bolognesi et al., 2008; Grossmann et al., 2009; Beermann et al., 2011). Similarly, Wg is a likely candidate for binding the Fz4 receptors in the developing appendages of Parasteatoda, although Wnt5, Wnt8, Wnt11-2, and $W n t 16$ are also expressed during limb bud formation and/or appendage elongation in this spider (Janssen et al., 2010). 


\section{The Function of Fz Receptors}

Despite the striking expression patterns of $f z$ genes in animals in many cases the specific functions of most $\mathrm{Fz}$ receptors remain enigmatic because knockout or knockdown appears to have no phenotypic consequences. This may be in part because these receptors have overlapping or possibly even redundant functions in certain contexts. For example, as described above, in Drosophila, $f z$ and $f z 2$ appear to have overlapping functions (Bhat, 1998; Kennerdell and Carthew, 1998; Chen and Struhl, 1999; Müller et al., 1999). Similarly in Tribolium knockdown of both $f z 1$ and $f z 2$ is required to induce germ band phenotypes and while RNAi against Tribolium $f z 1$ alone affects leg development, knockdown of either Tribolium $f z 2$ or $f z 4$ alone has no detectable effect (Beermann et al., 2011). Furthermore, Drosophila $f z 3$ mutants are viable and fertile and unlike $D f z 2$, this Drosophila $f z$ does not appear to function together with $f z 1$ (Sato et al., 1999).

It is also clear that $\mathrm{Fz}$ receptors can play more subtle roles perhaps to modulate Wnt signaling rather than transduce an all-or-nothing switch. For example $D f z 3$ is not a very effective transducer of $\mathrm{Wg}$ and may attenuate $\mathrm{Wg}$ activity, as well as receiving other Wnt ligands (Sato et al., 1999).

These findings are consistent with $\mathrm{Fz}$ receptors and Wnt ligands acting in complex combinatorial landscapes rather than simple linear pathways to modulate cell-to-cell communication to regulate gene expression, cell fate and ultimately development (van Amerongen and Nusse, 2009). It was previously shown in Parasteatoda and Tribolium that Wnt8 is required for the development of the SAZ and production of posterior segments (Bolognesi et al., 2008; McGregor et al., 2008). However, many other Wnts are expressed posteriorly in arthropods (Bolognesi et al., 2008; Janssen et al., 2010; Hayden and Arthur, 2014; Hayden et al., 2015), and our $f z$ expression analysis suggests that these ligands work through multiple Fz receptors including Fzl in and proximal to the SAZ in panarthropods.

Interestingly there is increasing evidence that $\mathrm{Fz}$ receptors may interact with other ligands and signaling pathways. For example, it was shown in vertebrates that $\mathrm{Fz} 4$ is bound by the Norrin ligand, which also antagonizes BMP signaling (Xu et al., 2004; Deng et al., 2013).

Therefore, $\mathrm{Fz}$ receptors are probably part of much larger integrated signaling landscapes in some developmental contexts. In order to fully understand the role as well as functional evolution of $f z$ genes in other panarthropods, systematic knockdown of these genes individually and in all combinations in a range of different developmental stages and tissues in models such as Parasteatoda, where functional tools are available (Hilbrant et al., 2012), is required.

\section{Conclusions}

We have shown that the panarthropod $f z$ genes show complex patterns of gene expression and divergence in the protein sequences that they encode. However, our analysis has allowed the identification of the possibly ancestral roles for some of these receptors in specific aspects of development such as Fz2 genes in segmentation in arthropods. Further disentangling the roles of these important receptors in animal development requires further comparative analysis of their expression and detailed dissections of their function in different animals and developmental contexts.

\section{Author Contributions}

This study was conceived by AM, MHi, and RJ. All authors contributed to the acquisition of the data. AM, MHi, RJ, and AS wrote the manuscript with contributions to drafts by MW, NT, GG, NP, and MHo. The final version of the manuscript was approved by all authors. All authors agreed to be accountable for all aspects of the work in ensuring that questions related to the accuracy or integrity of any part of the work are appropriately investigated and resolved.

\section{Acknowledgments}

We thank Oxford Brookes University for a Nigel Groome studentship for AS. This work was also supported by the Swedish Research Council. We also gratefully acknowledge the support of the New South Wales Government Department of Environment and Climate Change by provision of a permit SL100159 to collect onychophorans at Kanangra-Boyd National Park and to the Australian Government Department of the Environment, Water, Heritage and the Arts for export permits WT2009-4598 and WT2012-4704. We thank Jean Joss, Rolf Ericsson, Robyn Stutchbury and, especially, Noel Tait, for their help during onychophoran collection. We also acknowledge the DNA Microarray and Deep-Sequencing Facility, Transcriptomanalyselabor, UMG Göttingen for library preparation, sequencing, and total RNA extraction of Pholcus embryos.

\section{Supplementary Material}

The Supplementary Material for this article can be found online at: http://journal.frontiersin.org/article/10.3389/fevo. 2015.00096

Figure S1 | Multiple sequence alignment of all Frizzled sequences found in genomic resources for 10 selected panarthropod species including $D$. melanogaster.

Figure S2 | Maximum-likelihood tree inferred from the analysis of conserved regions in Frizzled sequences of 10 selected panarthropod species, including $D$. melanogaster.

Figure S3 | Additional aspects of Glomeris $f z 2$ and $f z 4$ expression. (A-C) Ventral views; anterior to the left, (D-G) dissected appendages, anterior views, $(\mathbf{H}-\mathbf{J})$ lateral views; anterior to the left. Arrows in $(\mathbf{H}-\mathbf{J})$ point to a sharp border of expression within the extraembryonic tissue. The outline of the embryos proper is indicated (dashed lines). The asterisk in $\mathbf{( H )}$ marks the border of expression in embryonic tissue. an, antenna; md, mandible; mx, maxilla; L walking limb; Ir, labrum; oc, ocular region; S, stomodaeum; saz, segment addition zone. 


\section{References}

Akiyama-Oda, Y., and Oda, H. (2003). Early patterning of the spider embryo: a cluster of mesenchymal cells at the cumulus produces Dpp signals received by germ disc epithelial cells. Development 130, 1735-1747. doi: 10.1242/dev. 00390

Beermann, A., Prühs, R., Lutz, R., and Schroder, R. (2011). A context-dependent combination of Wnt receptors controls axis elongation and leg development in a short germ insect. Development 138, 2793-2805. doi: 10.1242/dev.063644

Bhanot, P., Brink, M., Samos, C. H., Hsieh, J. C., Wang, Y., Macke, J. P., et al. (1996). A new member of the frizzled family from Drosophila functions as a wingless receptor. Nature 382, 225-230. doi: 10.1038/382225a0

Bhat, K. M. (1998). Frizzled and frizzled 2 play a partially redundant role in wingless signaling and have similar requirements to wingless in neurogenesis. Cell 95, 1027-1036. doi: 10.1016/S0092-8674(00)81726-2

Bolognesi, R., Farzana, L., Fischer, T. D., and Brown, S. J. (2008). Multiple Wnt genes are required for segmentation in the short-germ embryo of Tribolium castaneum. Curr. Biol. 18, 1624-1629. doi: 10.1016/j.cub.2008.09.057

Cao, Z., Yu, Y., Wu, Y., Hao, P., Di, Z., He, Y., et al. (2013). The genome of Mesobuthus martensii reveals a unique adaptation model of arthropods. Nat. Commun. 4, 2602. doi: 10.1038/ncomms3602

Castresana, J. (2000). Selection of conserved blocks from multiple alignments for their use in phylogenetic analysis. Mol. Biol. Evol. 17, 540-552. doi: 10.1093/oxfordjournals.molbev.a026334

Chen, C. M., and Struhl, G. (1999). Wingless transduction by the Frizzled and Frizzled2 proteins of Drosophila. Development 126, 5441-5452.

Chipman, A. D., Ferrier, D. E., Brena, C., Qu, J., Hughes, D. S., Schroder, R., et al. (2014). The first myriapod genome sequence reveals conservative arthropod gene content and genome organisation in the centipede Strigamia maritima. PLoS Biol. 12:e1002005. doi: 10.1371/journal.pbio.1002005

Cho, S. J., Valles, Y., Giani, V. C. Jr., Seaver, E. C., and Weisblat, D. A. (2010). Evolutionary dynamics of the Wnt gene family: a lophotrochozoan perspective. Mol. Biol. Evol. 27, 1645-1658. doi: 10.1093/molbev/msq052

Dann, C. E., Hsieh, J. C., Rattner, A., Sharma, D., Nathans, J., and Leahy, D. J. (2001). Insights into Wnt binding and signalling from the structures of two Frizzled cysteine-rich domains. Nature 412, 86-90. doi: 10.1038/350 83601

Darriba, D., Taboada, G. L., Doallo, R., and Posada, D. (2011). ProtTest 3: fast selection of best-fit models of protein evolution. Bioinformatics 27, 1164-1165. doi: 10.1093/bioinformatics/btr088

Deng, C., Reddy, P., Cheng, Y., Luo, C. W., Hsiao, C. L., and Hsueh, A. J. (2013). Multi-functional norrin is a ligand for the LGR4 receptor. J. Cell Sci. 126, 2060-2068. doi: $10.1242 /$ jcs. 123471

Dohle, W. (1964). Die embryonalentwicklung von Glomeris marginata (Villers) im vergleich zur entwicklung anderer diplopoden. Zool. Jahrb. Anat. 81, 241-310.

Edgar, R. C. (2004). MUSCLE: multiple sequence alignment with high accuracy and high throughput. Nucleic Acids Res. 32, 1792-1797. doi: 10.1093/nar/gkh340

Edgecombe, G. D., and Legg, D. A. (2014). Origins and early evolution of arthropods. Palaeontology 57, 457-468. doi: 10.1111/pala.12105

Eriksson, B. J., and Tait, N. N. (2012). Early development in the velvet worm Euperipatoides kanangrensis Reid 1996 (Onychophora: Peripatopsidae). Arthropod Struct. Dev. 41, 483-493. doi: 10.1016/j.asd.2012.02.009

Force, A., Lynch, M., Pickett, F. B., Amores, A., Yan, Y. L., and Postlethwait, J. (1999). Preservation of duplicate genes by complementary, degenerative mutations. Genetics 151, 1531-1545.

Gouy, M., Guindon, S., and Gascuel, O. (2010). SeaView version 4: a multiplatform graphical user interface for sequence alignment and phylogenetic tree building. Mol. Biol. Evol. 27, 221-224. doi: 10.1093/molbev/msp259

Grabherr, M. G., Haas, B. J., Yassour, M., Levin, J. Z., Thompson, D. A., Amit, I., et al. (2011). Full-length transcriptome assembly from RNA-Seq data without a reference genome. Nat. Biotechnol. 29, 644-652. doi: 10.1038/nbt.1883

Grossmann, D., Scholten, J., and Prpic, N. M. (2009). Separable functions of wingless in distal and ventral patterning of the Tribolium leg. Dev. Genes Evol. 219, 469-479. doi: 10.1007/s00427-009-0310-z

Hayden, L., and Arthur, W. (2014). The centipede Strigamia maritima possesses a large complement of Wnt genes with diverse expression patterns. Evol. Dev. 16, 127-138. doi: 10.1111/ede.12073
Hayden, L., Schlosser, G., and Arthur, W. (2015). Functional analysis of centipede development supports roles for Wnt genes in posterior development and segment generation. Evol. Dev. 17, 49-62. doi: 10.1111/ede.12112

Hilbrant, M., Damen, W. G., and McGregor, A. P. (2012). Evolutionary crossroads in developmental biology: the spider Parasteatoda tepidariorum. Development 139, 2655-2662. doi: 10.1242/dev.078204

Hogvall, M., Schonauer, A., Budd, G. E., McGregor, A. P., Posnien, N., and Janssen, R. (2014). Analysis of the Wnt gene repertoire in an onychophoran provides new insights into the evolution of segmentation. Evodevo 5:14. doi: 10.1186/2041-9139-5-14

Huang, H. C., and Klein, P. S. (2004). The Frizzled family: receptors for multiple signal transduction pathways. Genome Biol. 5:234. doi: 10.1186/gb-2004-5-7234

Janson, K., Cohen, E. D., and Wilder, E. L. (2001). Expression of DWnt6, DWnt10, and DFz4 during Drosophila development. Mech. Dev. 103, 117-120. doi: 10.1016/S0925-4773(01)00323-9

Janssen, R., and Budd, G. E. (2013). Deciphering the onychophoran 'segmentation gene cascade': gene expression reveals limited involvement of pair rule gene orthologs in segmentation, but a highly conserved segment polarity gene network. Dev. Biol. 382, 224-234. doi: 10.1016/j.ydbio.2013. 07.010

Janssen, R., Jörgensen, M., Lagebro, L., and Budd, G. E. (2015a). Fate and nature of the onychophoran mouth-anus furrow and its contribution to the blastopore. Proc. Biol. Sci. 282:20142628. doi: 10.1098/rspb.2014.2628

Janssen, R., Jorgensen, M., Prpic, N. M., and Budd, G. E. (2015b). Aspects of dorsoventral and proximo-distal limb patterning in onychophorans. Evol. Dev. 17, 21-33. doi: 10.1111/ede.12107

Janssen, R., Le Gouar, M., Pechmann, M., Poulin, F., Bolognesi, R., Schwager, E. E., et al. (2010). Conservation, loss, and redeployment of Wnt ligands in protostomes: implications for understanding the evolution of segment formation. BMC Evol. Biol. 10:374. doi: 10.1186/1471-2148-10-374

Janssen, R., and Posnien, N. (2014). Identification and embryonic expression of Wnt2, Wnt4, Wnt5 and Wnt 9 in the millipede Glomeris marginata (Myriapoda: Diplopoda). Gene Expr. Patterns 14, 55-61. doi: 10.1016/j.gep.2013.12.003

Janssen, R., Prpic, N. M., and Damen, W. G. (2004). Gene expression suggests decoupled dorsal and ventral segmentation in the millipede Glomeris marginata (Myriapoda: Diplopoda). Dev. Biol. 268, 89-104. doi: 10.1016/j.ydbio.2003.12.021

Kennerdell, J. R., and Carthew, R. W. (1998). Use of dsRNA-mediated genetic interference to demonstrate that frizzled and frizzled 2 act in the wingless pathway. Cell 95, 1017-1026. doi: 10.1016/S0092-8674(00)81725-0

Lawson, D., Arensburger, P., Atkinson, P., Besansky, N. J., Bruggner, R. V., Butler, R., et al. (2009). VectorBase: a data resource for invertebrate vector genomics. Nucleic Acids Res. 37, D583-D587. doi: 10.1093/nar/gkn857

Logan, C. Y., and Nusse, R. (2004). The Wnt signaling pathway in development and disease. Annu. Rev. Cell Dev. Biol. 20, 781-810. doi: 10.1146/annurev.cellbio.20.010403.113126

Lynch, M., and Force, A. (2000). The probability of duplicate gene preservation by subfunctionalization. Genetics 154, 459-473.

Mayer, G. (2006). Origin and differentiation of nephridia in the Onychophora provide no support for the Articulata. Zoomorphology 125, 1-12. doi: 10.1007/s00435-005-0006-5

McGregor, A. P., Pechmann, M., Schwager, E. E., Feitosa, N. M., Kruck, S., Aranda, M., et al. (2008). Wnt8 is required for growth-zone establishment and development of opisthosomal segments in a spider. Curr. Biol. 18, 1619-1623. doi: $10.1016 /$ j.cub.2008.08.045

Mittmann, B., and Wolff, C. (2012). Embryonic development and staging of the cobweb spider Parasteatoda tepidariorum C. L. Koch, 1841 (syn.: Achaearanea tepidariorum; Araneomorphae; Theridiidae). Dev. Genes Evol. 222, 189-216. doi: 10.1007/s00427-012-0401-0

Müller, H. A., Samanta, R., and Wieschaus, E. (1999). Wingless signaling in the Drosophila embryo: zygotic requirements and the role of the frizzled genes. Development 126, 577-586.

Murat, S., Hopfen, C., and McGregor, A. P. (2010). The function and evolution of Wnt genes in arthropods. Arthropod Struct. Dev. 39, 446-452. doi: 10.1016/j.asd.2010.05.007

Nielsen, C. (1995). Animal Evolution: Interrelationships of the Living Phyla. Oxford: Oxford University Press. 
Park, W. J., Liu, J., and Adler, P. N. (1994). Frizzled gene expression and development of tissue polarity in the Drosophila wing. Dev. Genet. 15, 383-389. doi: $10.1002 /$ dvg.1020150410

Pechmann, M., Khadjeh, S., Turetzek, N., McGregor, A. P., Damen, W. G., and Prpic, N. M. (2011). Novel function of Distal-less as a gap gene during spider segmentation. PLoS Genet. 7:e1002342. doi: 10.1371/journal.pgen.1002342

Posnien, N., Zeng, V., Schwager, E. E., Pechmann, M., Hilbrant, M., Keefe, J. D., et al. (2014). A comprehensive reference transcriptome resource for the common house spider Parasteatoda tepidariorum. PLoS ONE 9:e104885. doi: 10.1371/journal.pone.0104885

Prpic, N. M., Schoppmeier, M., and Damen, W. G. (2008). Whole-mount in situ hybridization of spider embryos. CSH Protoc. 2008:pdb.prot5068. doi: $10.1101 /$ pdb.prot5068

Prpic, N. M., and Tautz, D. (2003). The expression of the proximodistal axis patterning genes Distal-less and dachshund in the appendages of Glomeris marginata (Myriapoda: Diplopoda) suggests a special role of these genes in patterning the head appendages. Dev. Biol. 260, 97-112. doi: 10.1016/S00121606(03)00217-3

Prud'homme, B., Lartillot, N., Balavoine, G., Adoutte, A., and Vervoort, M. (2002). Phylogenetic analysis of the Wnt gene family. Insights from lophotrochozoan members. Curr. Biol. 12, 1395. doi: 10.1016/s0960-9822(02)01068-0

Richards, S., Gibbs, R. A., Weinstock, G. M., Brown, S. J., Denell, R., Beeman, R. W., et al. (2008). The genome of the model beetle and pest Tribolium castaneum. Nature 452, 949-955. doi: 10.1038/nature06784

Sato, A., Kojima, T., Ui-Tei, K., Miyata, Y., and Saigo, K. (1999). Dfrizzled-3, a new Drosophila Wnt receptor, acting as an attenuator of Wingless signaling in wingless hypomorphic mutants. Development 126, 4421-4430.

Schenkelaars, Q., Fierro-Constain, L., Renard, E., Hill, A. L., and Borchiellini, C. (2015). Insights into Frizzled evolution and new perspectives. Evol. Dev. 17, 160-169. doi: 10.1111/ede.12115

Sharma, P. P., Kaluziak, S. T., Perez-Porro, A. R., González, V. L., Hormiga, G., Wheeler, W. C., et al. (2014). Phylogenomic interrogation of arachnida reveals systemic conflicts in phylogenetic signal. Mol. Biol. Evol. 31, 2963-2984. doi: $10.1093 / \mathrm{molbev} / \mathrm{msu} 235$

Sievers, F., Wilm, A., Dineen, D., Gibson, T. J., Karplus, K., Li, W., et al. (2011). Fast, scalable generation of high-quality protein multiple sequence alignments using Clustal Omega. Mol. Syst. Biol. 7, 539. doi: 10.1038/msb.2011.75

Sivasankaran, R., Calleja, M., Morata, G., and Basler, K. (2000). The Wingless target gene $D f z 3$ encodes a new member of the Drosophila Frizzled family. Mech. Dev. 91, 427-431. doi: 10.1016/S0925-4773(99)00313-5

Stamatakis, A., Ludwig, T., and Meier, H. (2005). RAxML-III: a fast program for maximum likelihood-based inference of large phylogenetic trees. Bioinformatics 21, 456-463. doi: 10.1093/bioinformatics/bti191

Strutt, D. (2003). Frizzled signalling and cell polarisation in Drosophila and vertebrates. Development 130, 4501-4513. doi: 10.1242/dev.00695
Tauriello, D. V., Jordens, I., Kirchner, K., Slootstra, J. W., Kruitwagen, T., Bouwman, B. A., et al. (2012). Wnt/beta-catenin signaling requires interaction of the Dishevelled DEP domain and $\mathrm{C}$ terminus with a discontinuous motif in Frizzled. Proc. Natl. Acad. Sci. U.S.A. 109, E812-E820. doi: 10.1073/pnas.1114802109

Terrapon, N., Li, C., Robertson, H. M., Ji, L., Meng, X., Booth, W., et al. (2014). Molecular traces of alternative social organization in a termite genome. Nat. Commun. 5, 3636. doi: 10.1038/ncomms4636

Umbhauer, M., Diiane, A., Goisset, C., Penzo-Mendez, A., Riou, J. F., Boucaut, J. C., et al. (2000). The C-terminal cytoplasmic Lys-thr-X-X-X-Trp motif in frizzled receptors mediates Wnt/beta-catenin signalling. EMBO J. 19, 4944-4954. doi: 10.1093/emboj/19.18.4944

van Amerongen, R., and Nusse, R. (2009). Towards an integrated view of Wnt signaling in development. Development 136, 3205-3214. doi: 10.1242/dev.033910

Veeman, M. T., Axelrod, J. D., and Moon, R. T. (2003). A seconds canon. Functions and mechanisms of beta-catenin-independent Wnt signaling. Dev. Cell 5, 367-377. doi: 10.1016/S1534-5807(03)00266-1

Wolff, C., and Hilbrant, M. (2011). The embryonic development of the central American wandering spider Cupiennius salei. Front. Zool. 8:15. doi: 10.1186/1742-9994-8-15

Wong, H. C., Bourdelas, A., Krauss, A., Lee, H. J., Shao, Y., Wu, D., et al. (2003). Direct binding of the PDZ domain of dishevelled to a conserved internal sequence in the C-terminal region of Frizzled. Mol. Cell 12, 1251-1260. doi: 10.1016/S1097-2765(03)00427-1

Xu, Q., Wang, Y., Dabdoub, A., Smallwood, P. M., Williams, J., Woods, C., et al. (2004). Vascular development in the retina and inner ear: control by Norrin and Frizzled-4, a high-affinity ligand-receptor pair. Cell 116, 883-895. doi: 10.1016/S0092-8674(04)00216-8

Zeng, V., and Extavour, C. G. (2012). ASGARD: an open-access database of annotated transcriptomes for emerging model arthropod species. Database (Oxford) 2012:bas048. doi: 10.1093/database/ bas 048

Conflict of Interest Statement: The authors declare that the research was conducted in the absence of any commercial or financial relationships that could be construed as a potential conflict of interest.

Copyright (c) 2015 Janssen, Schönauer, Weber, Turetzek, Hogvall, Goss, Patel, McGregor and Hilbrant. This is an open-access article distributed under the terms of the Creative Commons Attribution License (CC BY). The use, distribution or reproduction in other forums is permitted, provided the original author(s) or licensor are credited and that the original publication in this journal is cited, in accordance with accepted academic practice. No use, distribution or reproduction is permitted which does not comply with these terms. 